\title{
Model scale investigation of the effect of different speed reduction strategies on cavitating propeller radiated noise
}

\author{
Giorgio Tani, Michele Viviani \\ Department of Electrical, Electronic, Telecommunication \\ Engineering and Naval Architecture, (DITEN), Genoa \\ University \\ Genoa, Italy
}

\author{
Enrico Rizzuto \\ Department of Civil, Chemical and Environmental \\ Engineering, (DICCA), Genoa University \\ Genoa, Italy
}

\begin{abstract}
The continuous development of design tools and analysis procedures has allowed propeller performances to increase progressively during years following more and more stringent design requirements . High propeller efficiency and avoidance of erosive cavitation are considered nowadays basic requests for a well designed propeller. However this may not be enough to meet the requirements of high added value ships such as passengers ships, mega yachts, research vessels, naval vessels etc. These kinds of ship need a propulsion system not only with good efficiency but also with low levels of vibrations and noise transmitted inside the ship and with low underwater radiated noise. Moreover, the above mentioned ships often work in a rather wide range of operational condition. Actually, the possibility to operate at reduced speed regards almost all kinds of ships, due to the increasing attention to fuel consumption and exhaust gas emissions, and the related tendency towards slow steaming. Speed reduction may be obtained in different ways. In the case of ships equipped with CPPs, in principle it may be achieved reducing RPM at constant pitch or reducing pitch at constant RPM; the latter is usually preferred when a power take off is present. This results in working conditions far from design ones for the propeller; Cavitation and related radiated noise may increase significantly in similar situations, despite the speed reduction is felt to be generally beneficial for the purpose. In this work, the effect of different speed reduction strategies on cavitation and radiated noise is studied by means of cavitation tunnel tests of four CPPs including both single screw and twin screw configurations.
\end{abstract}

Keywords-Cavitation, Radiated noise, Experimental fluid dynamics, Ships operations, $\mathrm{CP}$ propellers

\section{INTRODUCTION}

Propeller design has evolved significantly in last years, with the introduction of numerical methods which can provide an ever improving assessment of propeller characteristics, in non stationary and cavitating situations, corresponding not only to the design point, but also to off-design conditions. This assessment has become rather usual, with numerical methods being able to predict propeller characteristic curves (and cavitating behaviour) in correspondence to a wide range of advance coefficients. Nevertheless, problems still exist if conditions far from design" are considered, and if interest is posed on side effects of propeller cavitation, such as radiated noise. The ever increasing demand for improvement of propeller performances has moved attention of propeller designers to these latter problems, especially when dealing with "high added value ships" like for example cruise ships or megayachts, in which the comfort and noise related phenomena are of major interest. Moreover, underwater radiated noise problems are likely to gain more and more importance for all seagoing ships, due to the increased attention to environmental issues and in particular to the adverse effects of shipping noise on the marine fauna. This in the near future may lead to the formulation of requirements in terms of limits to the underwater noise radiation by ships, particularly in sensitive areas for marine animals. These requirements may regard design specifications and/or operative provisions: among the latter, speed reduction is often believed to be a practical means for reducing propeller excitation.

Slow steaming, on the other hand, has strong motivations also in the focus of Shipowners on the reduction of operating costs and, in part, on the need for limiting exhaust gas emissions. A reduction in speed, when dealing with ships equipped with CPPs, may be obtained in different ways, i.e. reducing pitch at constant RPM or reducing RPM at constant pitch. In many cases, the choice is not possible due to the particular ship propulsion plant, which may require to keep nearly constant RPM, because of the presence of a shaft generator run at constant speed. The effect of the alternative strategies on radiated noise may be very different, since the propeller cavitating behaviour is obviously remarkably different, with the presence of different phenomena.

In particular, pitch reduction at constant revolution rate is likely to produce cavitation on the pressure side of the blades and this kind of cavitation may generate rather high broadband noise levels, jeopardising the reduction in acoustic emission in comparison with full speed. The present paper digs into the subject of the gains obtainable in terms of underwater radiated noise by different strategies for speed reduction. 


\section{ROLE OF MODEL SCALE EXPERIMENTS}

The noise radiated by cavitating propellers at sea is a matter of interest for researchers since the second world war (for military reasons), but propeller radiated noise data are scarcely available in literature, right because of their "restricted" nature. Even less data are available when moving to off-design conditions such as reduced pitch of CPPs.

On the other hand, noise predictions by means of numerical tools are still rather difficult and computationally expensive, especially for what regards the continuous part of the spectrum.

As a consequence, model scale experiments are still largely adopted to study propeller radiated noise. The model scale approach however presents significant issues, too. Relevant scale effects act on cavitation, as for what regards the inception of the tip vortex [1], and on noise generation. Different scaling laws for radiated noise have been defined and discussed during years, some important examples are represented by the works of Baiter [2] and Strasberg [3]. A detailed analysis of the problem of noise scaling has been reported also by Blake in [4]. These studies allowed to develop suitable noise scaling laws and to obtain satisfactory results, as shown in [5] and [6].

Nevertheless experimental techniques and noise prediction procedures are still affected by significant uncertainties, thus the introduction of further experimental data is always beneficial, with the aim to increase the general understanding of the problem, in particular for what regards off-design conditions, for which literature data are even more scarce.

These data may be used in many different ways, they may be an useful source in general for the propeller designer, their systematic analysis may lead to the development of semiempirical methods for the prediction of propeller radiated noise and they may be used also for the validation of design and analysis codes.

Having this in mind, in the present paper results of a series of tests carried out during years at Genoa University Cavitation Tunnel on CPPs (namely two different propellers for a twin screw RORO-pax vessel and two propellers for two different single screw ships, i.e. a Research Vessel and a Costal Tanker) at different pitch settings are presented and reanalyzed, with a particular attention on the possible impact of different speed reduction strategies on cavitating propellers radiated noise.

The analysis of cavitation pattern and radiated noise for the two propellers of the RORO-pax vessel has already been presented in [7] focusing the attention on the design by optimization, which allowed to enhance significantly the propeller performances in two very different pitch settings.

In this work, the analysis is extended to the cases of two single screw vessels highlighting the effect of different speed reduction strategy for different ship configurations. Further, the complete hull wake is modelled in experiments for all tested propellers while in [7] the wake field was simulated only by means of shaft inclination and appendages.

This should allow to get a clearer insight into this topic and possibly a guidance for future ship operations considering possible and new stringent requirements.

\section{TEST CASES}

Four controllable pitch propellers are considered in the present work. The main characteristics of the propellers are reported in TABLE I. .

Propellers 1A and 1B (whose tests were carried out in the context of the project SILENV (Ship oriented Innovative soLutions to rEduce Noise and Vibrations [8]) are two different propellers for a twin screw RORO-pax vessels. In particular Propeller 1A is the real (original) ship propeller while Propeller 1B represents an alternative design obtained through an optimization procedure [9].

TABLE I. PROPELLER CHARACTERISTICS

\begin{tabular}{|l|l|l|l|l|}
\hline & Propeller 1A & Propeller 1B & Propeller 2 & Propeller 3 \\
\hline Number of blades & 4 & 4 & 4 & 4 \\
\hline Diameter [m] & 4.6 & 4.6 & 4.8 & 2.26 \\
\hline $\begin{array}{l}\text { Direction of } \\
\text { rotation }\end{array}$ & Right & Right & Left & Left \\
\hline $\begin{array}{l}\text { Design pitch ratio } \\
\text { at } 0.7 \mathrm{R}\end{array}$ & 1.08 & 1.106 & 0.87 & 0.94 \\
\hline
\end{tabular}

The optimization has been carried out with the aim of enhancing propeller cavitating behaviour at reduced pitch, limiting pressure side cavitation. The analysis of the performances of this propeller allows to evaluate to which extent a design optimisation may reduce the radiated noise in the mentioned situation.

Propeller 2 and Propeller 3 are the propellers of two single screw vessels, a Coastal Tanker and a Research Vessel respectively. Tests on these propellers have been carried out in the context of the EU project AQUO.

TABLE II. PROPELLER FUNCTIONING CONDITIONS

\begin{tabular}{|c|r|l|l|l|l|}
\hline \multicolumn{1}{|c|}{} & $\begin{array}{c}\text { Propeller } \\
\mathbf{1 a}\end{array}$ & $\begin{array}{c}\text { Propeller } \\
\mathbf{1 b}\end{array}$ & $\begin{array}{c}\text { Propeller } \\
\mathbf{2}\end{array}$ & $\begin{array}{c}\text { Propeller } \\
\mathbf{3}\end{array}$ \\
\hline \multirow{4}{*}{$\begin{array}{c}\text { Design } \\
\text { speed }\end{array}$} & Ship speed [Kn] & 24 & 24 & 15 & 11 \\
\cline { 2 - 6 } & Pitch ratio at 0.7R & 1.08 & 1.106 & 0.87 & 0.91 \\
\hline $\begin{array}{r}\text { Propeller } \\
\text { revolutions } \\
{[\mathrm{RPM}]}\end{array}$ & 180 & 180 & 120 & 203 \\
\hline $\begin{array}{c}\text { Low } \\
\text { speed at } \\
\text { constant } \\
\text { RPM }\end{array}$ & Ship speed [Kn] & 11 & 11 & 11 & 7 \\
\cline { 2 - 6 } & Pitch ratio at 0.7R & 0.47 & 0.47 & 0.521 & 0.464 \\
\hline $\begin{array}{r}\text { Propeller } \\
\text { revolutions } \\
{[\mathrm{RPM}]}\end{array}$ & 180 & 180 & 120 & 203 \\
\hline $\begin{array}{c}\text { Low } \\
\text { speed at } \\
\text { constant } \\
\text { pitch }\end{array}$ & Ship speed [Kn] & 11 & 11 & 11 & 7 \\
\cline { 2 - 6 } & Pitch ratio at 0.7R & 1.08 & 1.106 & 0.87 & 0.91 \\
\hline $\begin{array}{r}\text { Propeller } \\
\text { revolutions } \\
{[\mathrm{RPM}]}\end{array}$ & 83 & 83 & 84.6 & 0 \\
\hline
\end{tabular}

All the three considered ships are characterized by operational conditions at reduced speed, obtained by means of a pitch reduction at constant RPM. 
Due to the particular lay-out of their power plant, these ships cannot operate at different RPM. Nevertheless ideal working conditions obtained reducing the shaft revolutions at constant pitch have been derived from available data from self propulsion tests. The considered propeller functioning conditions at design speed and at low speed, (the latter obtained with both strategies: RPM control and pitch control), are summarized in TABLE II.

\section{EXPERIMENTAL SETUP}

Experiments were carried out at the Cavitation Tunnel facility of the Department of Electrical, Electronic, Telecommunication Engineering and Naval Architecture of the University of Genoa (DITEN); the layout of the tunnel is schematically represented in Fig. 1.

The facility is a Kempf\&Remmers closed water circuit tunnel with a squared testing section of $0.57 \mathrm{~m} \mathrm{x} 0.57 \mathrm{~m}$, having a total length of $2 \mathrm{~m}$.

The tunnel is equipped with a Kempf\&Remmers H39 dynamometer, which measures propeller thrust, torque and RPM. As usual, a mobile stroboscopic system allows to visualize cavitation phenomena on the propeller blades. Moreover, cavitation phenomena visualization in the testing section is also obtained by means of Allied Vision Tech Marlin F145B2 Firewire Cameras, with a resolution of 1392 x 1040 pixels and a frame rate up to $10 \mathrm{fps}$.

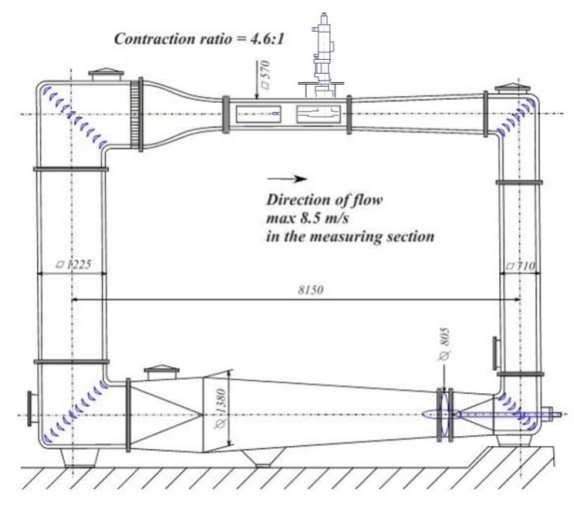

Fig. 1. DITEN cavitation tunnel

The Cavitation Tunnel is also equipped with a Laser Doppler Velocimetry (LDV) for non intrusive velocity field measurements.

Moreover, pressure sensors and hydrophones are used for the measurement of pressures and noise induced by the propeller. In present work, radiated noise measurements were carried out by means of Reson hydrophone TC4013, coupled with a Bruel and Kjaer 2635 charge amplifier.

As recommended by ITTC 2008, during all tests, oxygen content is constantly monitored by means of ABB dissolved oxygen sensor model 8012/170, coupled to an ABB AX400 analyser. In the majority of tests, oxygen content was kept constant and equal to $40 \%$ of saturation value at atmospheric pressure. Higher values however were considered when deemed necessary for a correct stimulation of cavitation (e.g. in functioning conditions with low depressurization levels, in correspondence to high values of the cavitation index).

For all the propellers, the influence of the wake has been taken into account carrying out experiments behind suitably built wire screens. In particular, the model scale nominal wake field was considered for the RORO-pax, while for the single screw ships the full scale nominal wake fields, evaluated by means of direct RANS computations, have been modelled experimentally.

Finally, for Propellers 1A, 1B and 2 the wake field, characterized by a significant vertical velocity component, has been simulated by means of the shaft inclination. The shaft inclination is $9^{\circ}$ for the RORO-pax propellers and $8.5^{\circ}$ for the Coastal Tanker; in the case of propeller 3 , the vertical component of flow was simulated suitably modifying the longitudinal wake to account for the difference in the inflow velocity.

Radiated noise measurements have been carried with the hydrophone mounted on a small cylindrical support protruding from a fin immersed in the tunnel flow, outside the propeller slipstream.

All tests carried out at Cavitation tunnel were carried out at constant propeller revolution rate, $(25 \mathrm{~Hz})$, except tests on Propeller 2 at reference pitch, carried out with a rate of 22.5 $\mathrm{Hz}$.

Propeller loading conditions are reproduced in terms of thrust coefficient and cavitation index, computed with the propeller revolution rate as in (1):

$$
\sigma_{N}=\frac{p_{0}-p_{v}}{1 / 2 \rho N^{2} D^{2}}
$$

In all cases, the net radiated noise is presented. The background noise is measured replacing the propeller with a dummy hub and reproducing the same operational conditions in the cavitation tunnel. Later, this recorded background noise spectrum is logarithmically subtracted to total noise spectrum measured in the presence of the operating propeller. The operation is carried out at frequencies for which the signal to noise ratio exceeds $3 \mathrm{~dB}$, otherwise $3 \mathrm{~dB}$ are simply subtracted to the signal (and a dotted line is presented).

Since hydrophone/propeller relative positions for the different experimental campaigns were slightly different, the net noise spectra are further scaled to the reference distance of one meter by a conventional spherical propagation law.

In this respect it has to be remarked that noise propagation inside the cavitation tunnel is rather different than the mentioned spherical model. Transfer functions for the cavitation tunnel should be considered in order to correctly predict free field radiation characteristics of the propellers.

This implies to measure the tunnel frequency response, that allows to consider the effect of the acoustical surrounding represented by the tunnel on the noise perceived by the hydrophone. This is performed adopting an underwater noise transducer whose free field emitting characteristics have been 
previously measured through dedicated tests, and acquiring the same signal in the tunnel in order to evaluate its effect. The complete procedure is described in [10].

Actually transfer functions of the UNIGE cavitation tunnel have been measured for tests on Propeller 2 and Propeller 3, as reported in [10], but they are not considered in this work, being not available for tests on the RORO-pax propellers.

On the other hand, aim of this work is to analyse the effect of speed reduction strategies on single propellers, not to compare performances of different propellers: for a given propeller, the effects of propagation in different operating conditions are likely to be the same, therefore not affecting the comparison between them.

Finally, full scale noise spectra are predicted according to the ITTC1987 [11] recommendations.

The experimental campaign at the Cavitation Tunnel, for all propellers (at two pitches: design and reduced), consisted of the activities reported in the following list.

a) Surveys of the inception of different cavitation phenomena;

b) Cavitation extension observation;

c) Radiated noise measurements;

d) Pressure pulses measurements;

In the following sections, results of cavitation tests and radiated noise measurements are presented, focusing the attention on cavitation extensions (and in particular on the different cavitation typologies in different functioning conditions) and the resulting radiated noise spectra.

\section{TEST RESULTS}

\section{A. Cavitation extensions}

The cavitation patterns on the propeller blades have been measured and photographed for a large number of working points (different combinations of thrust coefficient and cavitation index). Attention is focused on photographs collected for the design speed and the two different speed reduction strategies.

\section{Propeller $1 A$}

In Fig.2 and Fig.3, cavitation observations for Propeller 1A are reported.

As it can be seen, at the design speed Propeller 1A is characterized by significant suction side sheet cavitation and tip vortex cavitation at $0^{\circ}$ and $90^{\circ}$. This behaviour is in accordance with the presence of the wake disturbance at the disk top and with the effect of shaft inclination. The low speed condition obtained modifying the propeller pitch is, as expected, characterized by significant vortices at the pressure side leading edge in almost all angular positions. Finally, the third condition shows no cavitation, in accordance with the fact that the propeller works with its optimum pitch setting and, most important, with a lower revolution rate, hence an higher cavitation number.
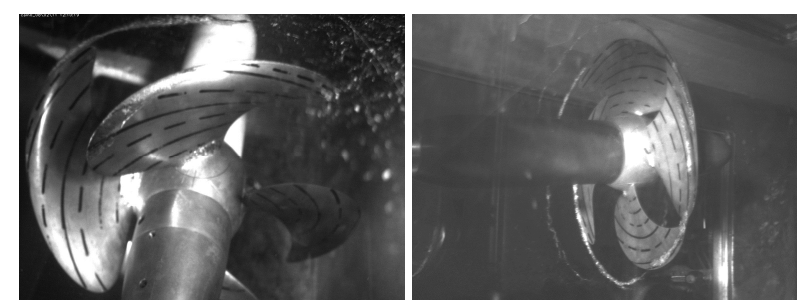

Fig. 2. Propeller 1A: Design speed, view from above (left); Speed reduction at constant RPM, view from starboard (right)

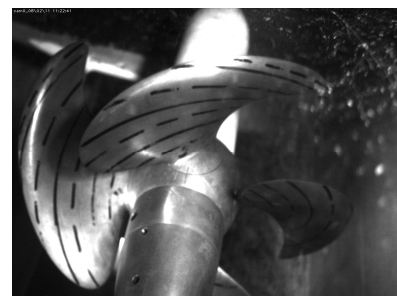

Fig. 3. Propeller 1A: Speed reduction at constant pitch, view from above

\section{Propeller $1 B$}

Similar results are presented in Fig. 4 and Fig. 5 for the optimized propeller 1B.
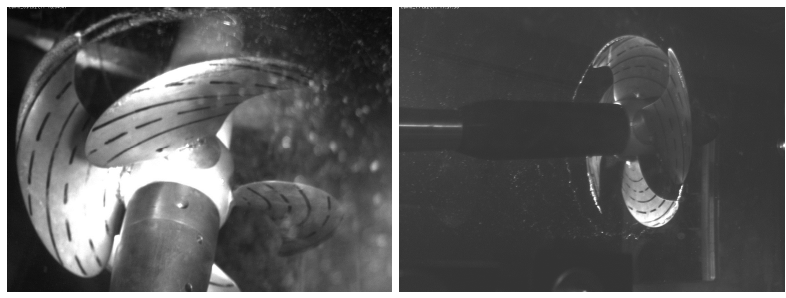

Fig. 4. Propeller 1B: Design speed, view from above (left); Speed reduction at constant RPM, view from starboard (right)

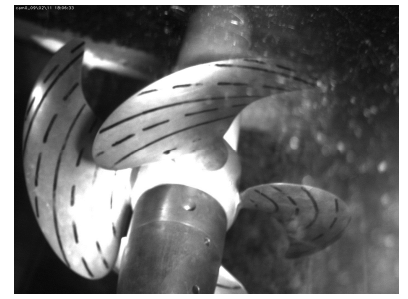

Fig. 5. Propeller 1B: Speed reduction at constant pitch, view from above

As it can be seen, the optimized propeller presents cavitation patterns qualitatively similar to those observed for Propeller 1A. In particular, suction side cavitation at design speed is slightly increased, with the presence of a second vortex detaching from the sheet cavity trailing edge at about $90^{\circ}$. Pressure side cavitation at reduced pitch is slightly reduced, (as expected, being this one of the aims for the optimization procedure. Leading edge pressure side vortices are however still present and well visible at all blade angular positions, suggesting that in such off-design conditions it was not possible to obtain a complete deletion of cavitation only 
acting on propeller geometry. Finally, the condition corresponding to the speed reduction at constant pitch is once again not interested by cavitation.

\section{Propeller 2}

Cavitation patterns observed for the Propeller 2 are rather similar to those already seen, despite the change from a twin screw to a single screw configuration. Suction side cavitation at design speed is seen in correspondence to the wake peak at 12 o' clock, with vortices persisting also at $90^{\circ}$ and $180^{\circ}$. In the reduced pitch condition, pressure side vortices are present, even if somehow intermittent.
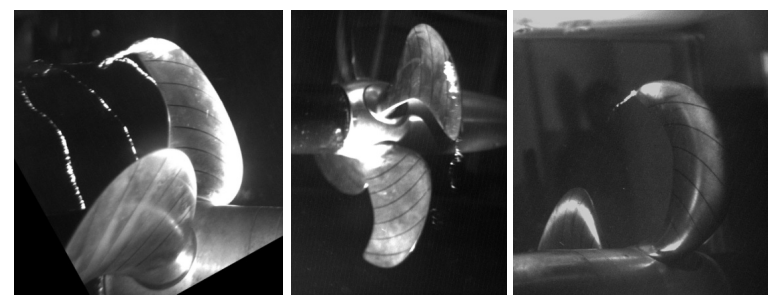

Fig. 6. Propeller 2: Design speed, $0^{\circ}$ view from starboard (left); Speed reduction at constant $\mathrm{RPM}, 270^{\circ}$ view from starboard (centre), $0^{\circ}$ view from portside (right)
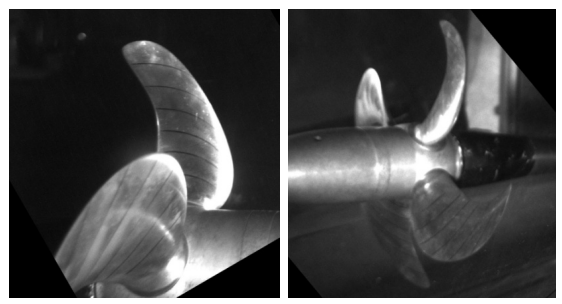

Fig. 7. Propeller 2: Speed reduction at constant pitch, $0^{\circ}$ view from starboard (left), view from portside (right)

Cavitation occurs mainly at $270^{\circ}$, due to the shaft inclination. Moreover a thin suction side vortex is present at $0^{\circ}$, still due to the effect of the wake peak, despite the considerable pitch reduction. Also in this case, revolution rate reduction at constant pitch leads to a cavitation free condition.

\section{Propeller 3}

In general cavitation extensions for Propeller 3 are rather limited, due to the rather low propeller loading. Keeping this in mind, results obtained for the three loading conditions, shown in Fig. 8 and Fig. 9, are absolutely in line with those shown for the other propellers, with suction side and pressure side cavitation respectively at design condition and at reduced pitch condition, and with no cavitation when speed reduction is achieved by means of RPM reduction at constant pitch.

The analysis of the test results above presented suggests that the effect of different speed reduction strategies may be more or less evident depending on the propeller load (at the design speed) and on the entity of the speed reduction. For example, the RORO-pax propellers feature a higher design speed, a higher load on the blades and also a larger reduction in speed. Accordingly, cavitation phenomena observed for propellers $1 \mathrm{~A}$ and $1 \mathrm{~B}$ are more developed than those of propellers 2 and 3, especially for what regards the reduced speed.
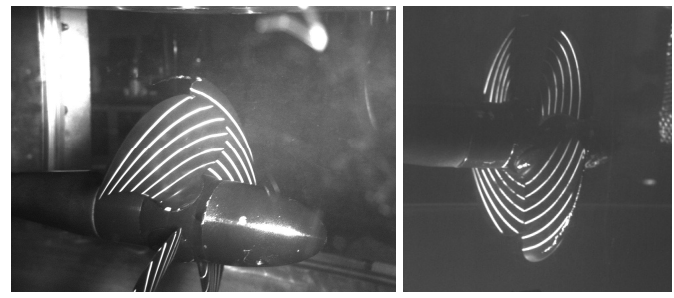

Fig. 8. Propeller 3: Design speed, view from starboard (left); Speed reduction at constant RPM, view from starboard

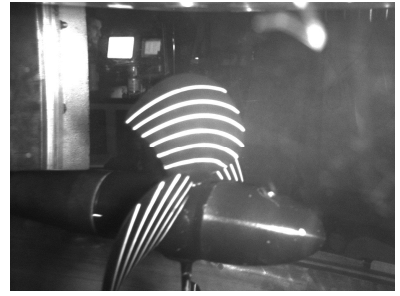

Fig. 9. Propeller 3: Speed reduction at constant pitch, view from starboard

The same trend, however, is evident in all cases: reducing the speed at constant RPM by only acting on propeller pitch causes the propeller to work in a condition far from the design, one, with negative angles of attack on a significant part of the blades. In addition, reducing the pitch while maintaining constant shaft revolutions, implies that the cavitation index keeps the same value featured at the design speed; as a consequence, a significant pressure side cavitation occurs. On the contrary, reducing the speed by decreasing only the shaft rotational speed makes the cavitation index increase even in the proximity of the design point. This leads to situations with very limited or no cavitation at all.

It has to be remarked, on the other hand, that the absence of cavitation for these conditions in model scale may also be partially due to scale effects. In particular the inception of tip vortex cavitation in full scale may occur at higher cavitation indices than in model scale, following the well known Mc Cormick law [1]. Further analyses are needed to quantify these effects. The possible presence of a weak tip vortex, anyway, should not increase significantly the radiated noise spectra, and therefore not affect the results presented below in terms of comparison between speed reduction strategies.

\section{$B$ Radiated noise}

In this paragraph full scale radiated noise spectra predicted for the four propellers in different working conditions are presented. Sound pressure power spectral densities in a narrowband representation are reported.

In the plots, the dotted lines represent those portions of the spectra for which the signal to noise ratio was lower than $3 \mathrm{~dB}$. As a consequence, these parts of the spectra need to be considered as an upper limit for the real propeller noise and not as a consistent noise prediction. This means that in the 
presence of dotted lines the real propeller noise levels are not known but they are anyway lower than the dotted line itself.

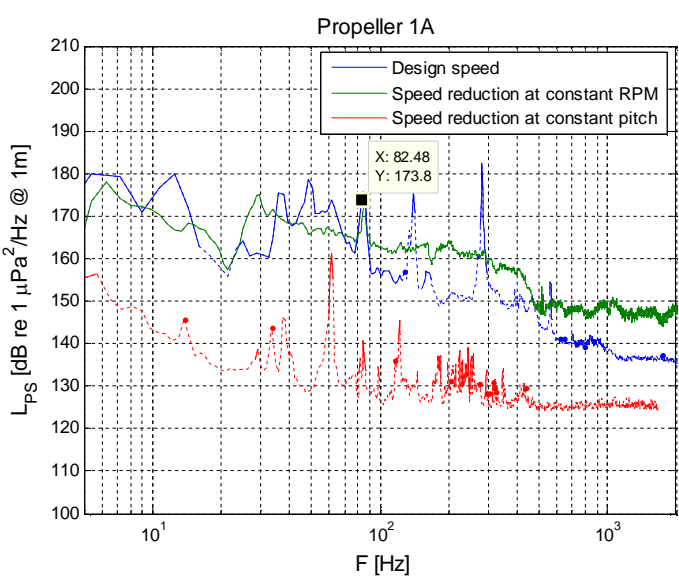

Fig. 10. RORO-pax Propeller 1A full scale radiated noise spectra

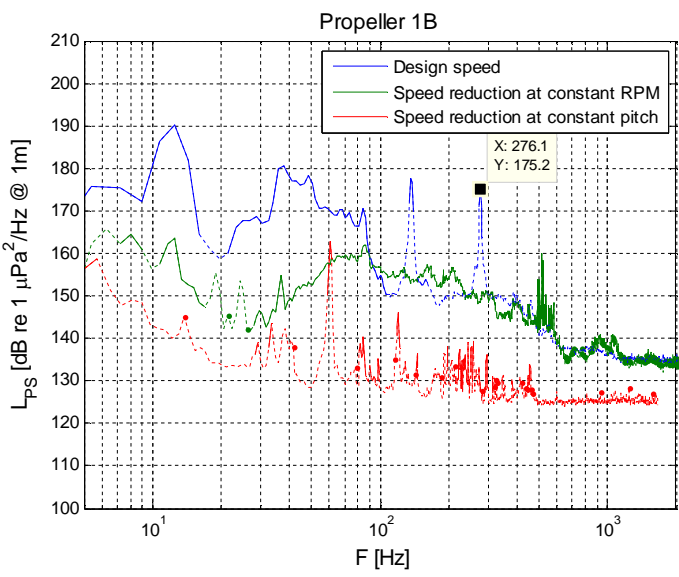

Fig. 11. RORO-pax Propeller 1B full scale radiated noise spectra

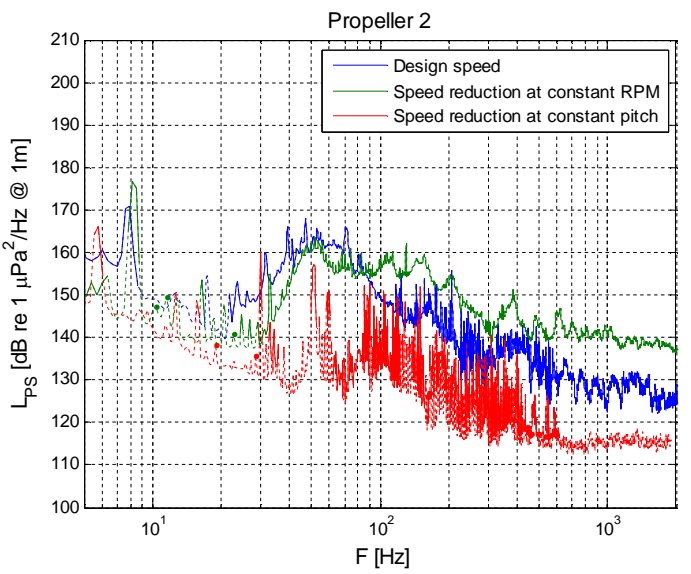

Fig.12. Coastal tanker propeller 2 full scale radiated noise spectra

In general, when analysing the spectra, attention should be focused on the continuous part of the spectrum, which is the part associated to cavitation. Components at the blade rate are also present, however, due to the small distance between the hydrophone and the propeller. In this situation, it is difficult to separate the near field pressure fluctuations caused by blade passages.

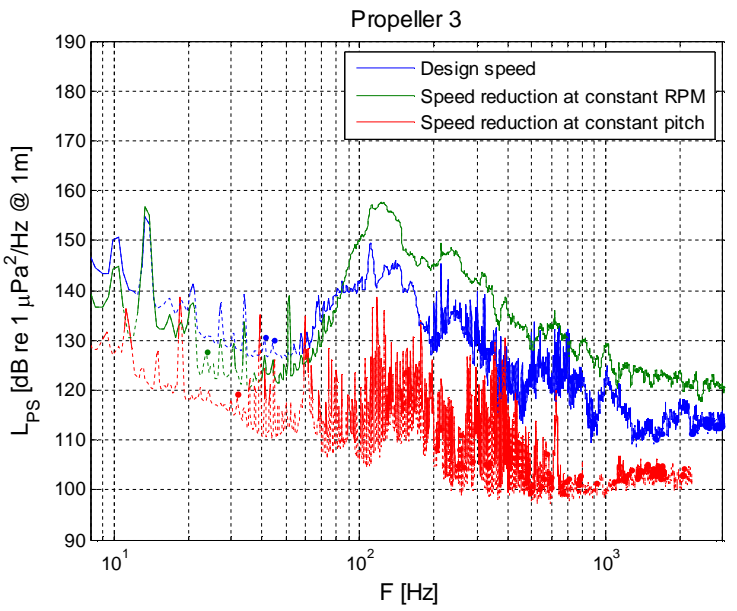

Fig. 13. Research Vessel propeller 3 full scale radiated noise spectra

A certain number of tonal components is present at higher frequencies, especially for conditions with limited cavitation and low noise. These components are likely to be associated with structural vibrations of the tunnel and mechanical noise from the dynamometer: in fact, they both have not been designed for the purpose of noise measurements; this does not prevent anyway a complete analysis of the effect of different speed reduction strategies, which is the aim of the present work. In case full scale noise predictions are needed, suitable techniques need to be adopted in order to alleviate these problems.

Finally, two rather high tonal components are observed for both RORO-pax propellers at design speed. These components are probably generated by vibration of the wire screen adopted for the wake modelling. The flow speed for these working conditions is actually close to the upper limit achievable with wire screens inside the tunnel.

At design speed, all considered propellers show typical suction side cavitation spectra, characterized by a medium low frequency hump, more or less intense depending on propeller dimensions, operating conditions and cavitation extensions. These spectra are in general of the same order and in some cases lower than those measured for the reduced speed in constant RPM conditions.

The latter conditions (pitch reduction) are all characterized by significant medium high frequency broadband noise, following the typical spectrum generated by pressure side leading edge vortices. Despite the apparently limited cavitation extensions observed, these spectra exceed design speed spectra for Propellers 3 and for Propeller 2 exception made for the frequency range $20 \div 80 \mathrm{~Hz}$. As regards the RORO-pax Propeller $1 \mathrm{~A}$, noise at reduced pitch is again higher than the one at design speed at medium high frequency, while the two spectra are comparable at low frequencies. These results are not surprising, considering the observed cavitation extensions: 
pressure side cavitation is actually known to produce higher noise levels than suction side tip vortices and sheet cavitation.

It has to be stressed that, reducing the ship speed at constant RPM leads to such high noise levels despite a remarkable speed reduction of about $13 \mathrm{Kn}$.

Finally, the radiated noise spectra measured for Propeller 1B allow to appreciate the effects of an optimised design. In this case, noise at design pitch is almost unvaried, only slightly increased in the frequency range corresponding to the tip vortex cavitation noise. On the other hand, noise at reduced pitch is significantly lower. Differences are of about $10 \mathrm{~dB}$, with even larger values at low frequencies. This result may be considered very satisfactory, considering the very different design points to be addressed in this case. However, it is clear that, even with an optimised propeller, the reduction of radiated noise is not comparable to the one which can be obtained with a speed reduction at constant pitch.

As a matter of fact, for all the propellers spectra here reported, the conditions corresponding to a speed decrease by revolution reduction at constant pitch provide lower levels than the corresponding conditions at constant RPM by about $20 \mathrm{~dB}$ or more, following that these conditions are characterized by no cavitation, as reported in previous paragraph.

The propeller noise was actually in most cases below the background noise of the tunnel and, as a consequence, for such conditions it was possible to define only an upper envelope for propeller noise levels, the actual ones remaining unknown. Nevertheless, these curves allow to appreciate the remarkable noise reduction achievable operating at constant pitch in comparison with pitch reduction

These results suggest that, from the point of view of radiated noise, the two considered speed reduction strategies imply quite different noise levels. When the speed reduction is performed decreasing propeller pitch at constant revolution rate, noise levels are (at least in correspondence to a part of the spectrum) even higher than those radiated at design speed, while much lower levels are obtained, on the contrary, if revolutions are lowered at constant pitch.

As demonstrated in the case of the optimized propeller, the improvements in terms of cavitation control when modifying the pitch achievable by optimising the propeller geometry are significant, but still the performances obtained by a RPM reduction at constant pitch are better.

\section{CONCLUSIONS}

The effects on cavitation and radiated noise of different combinatory laws for the speed reduction have been studied analysing the cases of three different ships and four propellers by means of extensive cavitation tunnel model scale experiments. In particular this work has focussed on the extreme strategies, i.e. pitch reduction at constant revolution rate and RPM reduction at constant pitch, without considering intermediate solutions.

The resulting working conditions of the propellers are completely different: when controlling the pitch, blade profiles operate at angles of attack far from the design ones, while, acting only on propeller RPM, the kinematic conditions keep similar to those for which the profiles are designed. In addition to this, a reduction in propeller RPM implies an increase in the cavitation number, while pitch reduction at constant RPM keeps constant the cavitation index.

Cavitation tunnel observations confirmed that these different working conditions result in completely different cavitation patterns. Reduced pitch conditions are all affected by pressure side cavitation, mainly consisting in pressure side leading edge vortices. This kind of cavitation is maybe not the most dangerous from the viewpoint of propeller erosion, or thrust breakdown, but its presence affects significantly the propeller performances in terms of pressure pulses and radiated noise.

This has been confirmed by cavitation tunnel noise measurements: the tests showed that reduced pitch conditions may cause the propeller to radiate even higher levels than at design speed.

On the contrary, conditions corresponding to the same reduced speed obtained reducing only the shaft speed showed no cavitation during model scale experiments. This resulted in very low noise emissions.

Finally in the paper a propeller designed by means of an optimization procedure was considered, too. The optimization procedure was intentionally aimed at reducing cavitation and related noise for off-design conditions at reduced pitch. From this point of view the goal of the optimization was achieved, cavitation extensions were reduced and noise levels were lowered by about $10 \mathrm{~dB}$. These results, even if remarkable, are however still not as effective as the adoption of the RPM control strategy for the ship speed reduction.

In conclusion, this work confirms that, from the point of view of cavitation and radiated noise, the common practice of reducing speed acting on propeller pitch at constant RPM is likely to cause a general worsening of propeller performances. This operational procedure is, however, largely adopted in the shipping industry, being often associated to the adoption of a simple and economic power plant for the ship, with PTO at constant RPM. The definition of the propulsion plant characteristics results therefore to be linked to underwater noise emissions, too. This, in the future, may contribute to push towards different choices in the plant characteristics, favoured, on the other hand, by the development of more flexible solutions for the power take off system

\section{AKNOWLEDGMENTS}

Part of this work was developed in the frame of the collaborative project AQUO (Achieve QUieter Oceans by shipping noise footprint reduction), funded by the European Commission within the Call FP7 SST.2012.1.1-1: Assessment and mitigation of noise impacts of the maritime transport on the marine environment, Grant agreement no 314227, coordinated topic "The Ocean of Tomorrow".

In addition part of present work has been carried out in the framework of the collaborative Project SILENV (Ships oriented Innovative soLutions to rEduce Noise \& Vibrations), 
financed by the European Commission under the $7^{\text {th }}$ Framework program, Grant agreement no 234182.

The content of this paper does not reflect the official opinion of the European Union. Responsibility for the information and views expressed in the paper lies entirely with the authors.

\section{REFERENCES}

[1] McCormick Jr., B.W.: "On Cavitation Produced by a Vortex Trailing From a Lifting Surfaces.", Journal of Basic Engineering, September 1962

[2] Baiter, H.J. “Aspects of cavitation noise.”, High Powered Propulsion of Large Ships Symp., NSMB, Wageningen, 1974.

[3] Strasberg, M. "Propeller cavitation noise after 35 years of study." Symp. of Noise and Fluids Eng, Proc ASME, Atlanta.

[4] Blake, W.K. "Propeller Cavitation Noise: The Problem of Scaling and Prediction.", ASME Symposium on cavitation and Multiphase Flow Noise (1986).

[5] Bark, G.: "Prediction of Propeller Cavitation Noise From Model Tests and Its Comparison With Full Scale Data.”, Transaction of the ASME, Journal of Fluids Engineering, Vol.107 (112-120), 1985
[6] Tani, G, Viviani, M., Gaggero, T., Rizzuto, E., "Single screw ships radiated noise measurements in model and full scale", Fourth International Symposium on Marine Propulsorssmp'15, Austin, Texas, USA, June 2015.

[7] Bertetta, D., Brizzolara, S., Gaggero, S., Savio, L. and Viviani, M. (2011). Experimental characterization of two CP propellers at different pitch settings, considering cavitating behaviour and related noise phenomena. Second International Symposium on Marine Propulsors, SMP 2011, Hamburg, Germany, June 2011

[8] www.silenv.eu

[9] Bertetta, D., Brizzolara, S., Gaggero, S., Viviani, M., Savio, L., "CPP propeller cavitation and noise optimization at different pitches with panel code and validation by cavitation tunnel measurements.", Ocean Engineering, 53: 177-195, 2012.

[10] Tani, G., Viviani, M., Armelloni, E., Nataletti, M. (2014) "Cavitation Tunnel Acoustic Characterisation and Application to Model Propeller Radiated Noise Measurements at Different Functioning Conditions", Proceedings of the Institution of Mechanical Engineers, Part M: Journal of Engineering for the Maritime Environment, (accepted, in print); ISSN: 1475-0902, SAGE Publishings.

[11] ITTC Cavitation Committee. Final report and recommendations to the 18th ITTC, Proceedings of the 18th (ITTC) International Towing Tank Conference, Kobe, Japan, 1987. 